\title{
Biobanking of fresh-frozen endoscopic biopsy specimens from esophageal adenocarcinoma
}

\author{
J. Stiekema, ${ }^{1}$ A. Cats, ${ }^{2}$ H. Boot,${ }^{2}$ A. M. J. Langers, ${ }^{3}$ O. Balague Ponz,${ }^{4}$ M. L. F. van Velthuysen, ${ }^{4}$ \\ L. M. Braaf, ${ }^{5}$ M. Nieuwland, ${ }^{6}$ J. W. van Sandick ${ }^{1}$ \\ ${ }^{1}$ Departments of Surgery, ${ }^{2}$ Gastroenterology and Hepatology, ${ }^{4}$ Pathology, ${ }^{5}$ Core Facility Molecular Pathology \\ and Biobanking, ${ }^{6}$ Deep Sequencing Facility, The Netherlands Cancer Institute, Amsterdam, and ${ }^{3}$ Department of \\ Gastroenterology and Hepatology, Leiden University Medical Center, Leiden, The Netherlands
}

\begin{abstract}
SUMMARY. The process of preparing endoscopic esophageal adenocarcinoma samples for next-generation DNA/RNA sequencing is poorly described. Therefore, we assessed the feasibility and pitfalls of preparing esophageal adenocarcinoma endoscopic biopsies toward DNA/RNA samples suitable for next-generation sequencing. In this prospective study, four tumor biopsy samples were collected from consecutive esophageal cancer patients during esophagogastroduodenoscopy and fresh-frozen in liquid nitrogen. DNA and RNA were isolated from samples with a tumor percentage of at least $\mathbf{5 0} \%$. For next-generation sequencing, double-stranded DNA (dsDNA) is required and high-quality RNA preferred. The quantity dsDNA and RNA quantity and quality were assessed with the Nanodrop 2000 spectrophotometer (Thermo Fisher Scientific, Waltham, MA, USA) and Agilent 2100 Bioanalyzer (Agilent, Santa Clara, CA, USA). Biopsy samples of 69 consecutive patients with esophageal adenocarcinoma were included. In five patients $(\mathbf{7} \%)$, the tumor percentage was less than $\mathbf{5 0} \%$ in all four biopsies. Using a protocol allowing simultaneous DNA and RNA isolation, the median dsDNA yield was $2.4 \mu \mathrm{g}$ (range 0.1-12.0 $\mu \mathrm{g}$ ) and the median RNA yield was $0.5 \mu \mathrm{g}$ (range $0.01-2.05 \mu \mathrm{g}$ ). The median RNA integrity number of samples that were fresh-frozen within 30 minutes after sampling was 6.7 (range 4.2-8.9) compared with $2.5(1.8-4.5)$ for samples that were fresh-frozen after 2 hours. The results from this study show that obtaining dsDNA and RNA for next-generation sequencing from endoscopic esophageal adenocarcinoma samples is feasible. Tumor percentage and dsDNA/RNA yield and quality emphasize the need for sampling multiple biopsies and minimizing the delay before fresh-freezing.
\end{abstract}

KEY WORDS: adenocarcinoma, DNA, endoscopy, translational research.

\section{INTRODUCTION}

The last decade of biomedical science is characterized by major technological advances. The increasing availability of tissue-based microarrays and, more recently, sequencing technology enable us to analyze tissue genome and transcriptome with unprecedented speed at relatively low costs. ${ }^{1}$ Because of these advances, it is likely that large-scale DNA and RNA analyses of tumor samples will be incorporated not only in future research but also in clinical practice. High-quality preserved tissue in combination with

Address correspondence to: Dr Johanna W. van Sandick, MD, $\mathrm{PhD}$, Department of Surgery, The Netherlands Cancer Institute, Plesmanlaan 121, 1066 CX Amsterdam, The Netherlands. Email: j.v.sandick@nki.nl

Financial support: Cornelis Vrolijk Development Fund.

Conflicts of interest: None declared.

1100 well-documented clinical data is considered a prerequisite for the generation of reliable data. Earlier papers on tissue collection and biobanking have mainly focused on the methodology of biobanking using surgical resection specimens..$^{2-5}$ Currently, the standard potentially curative treatment of esophageal cancer consists of neoadjuvant chemo(radio)therapy followed by surgical resection. ${ }^{6,7}$ The collection of untreated tumor samples is therefore limited to endoscopic biopsies. The small size of these biopsies poses a challenge in obtaining sufficient amounts of highquality DNA and RNA. Several studies on gene expression profiling of esophageal cancer using endoscopic biopsies have been published. ${ }^{8-12}$ However, detailed descriptions of sample collection and work-up are lacking in current literature. Such descriptions are invaluable in guiding biobanking initiatives and future experimental study design in 
esophageal cancer. The aim of the current study was to assess the feasibility of preparing untreated esophageal adenocarcinoma endoscopic biopsies toward DNA and RNA samples suitable for next-generation sequencing. This information is important as it can help in guiding future and ongoing biobanking initiatives that are essential for studies aimed at genomically characterizing this often-lethal cancer.

\section{METHODS}

\section{Patients}

In September 2008, our prospective study started with collecting endoscopic biopsies of esophageal cancer for genomic profiling. Patients with esophageal cancer who presented with potentially curable disease were eligible for the study. In the current study, only patients with esophageal adenocarcinoma who were planned to undergo neoadjuvant chemoradiotherapy followed by surgical resection were included. Clinical patient data were collected in a prospectively maintained database. Biopsy samples were collected after oral and written informed consent was obtained. The study was approved by the ethical committee.

\section{Sample collection}

During endoscopy, four tumor biopsy samples and two biopsy samples from normal esophageal tissue were obtained using a 2.2-mm biopsy forceps. The samples were placed on gauze with $\mathrm{NaCl} 0.9 \%$ to prevent dehydration, and preferably immediately transported to the pathology department and freshfrozen in liquid nitrogen. The time of fresh-freezing was recorded. All samples were stored at $-80^{\circ} \mathrm{C}$ until further processing.

\section{Tumor percentage}

The tumor percentage in each biopsy sample was estimated by the following method: using a cryostat at $-20^{\circ} \mathrm{C}$ up to 20 slides of $30-\mu \mathrm{m}$ thickness were prepared and immediately stored at $-80^{\circ} \mathrm{C}$. Before and after these 20 slides, an $8-\mu \mathrm{m}$ slide was prepared for hematoxylin and eosin staining. A pathologist scored the tumor percentage in both $8-\mu \mathrm{m}$ slides. The average of the tumor percentage in both $8-\mu \mathrm{m}$ slides was considered the tumor percentage in the biopsy sample. When the average tumor percentage was below $50 \%$, a second biopsy sample was processed, and so on until a sample with a tumor percentage of at least $50 \%$ was found. In biopsy samples with a clear separation of normal and tumor-rich areas, manual microdissection was performed to enrich the tumor percentage. We did not perform laser capture microdissection in this study.

(C) 2015 International Society for Diseases of the Esophagus

\section{DNA and RNA extraction and quality control}

Because of the limited availability of tumor tissue, DNA and RNA were simultaneously extracted from every sample using the DNA/RNA AllPrep microkit (Qiagen, Basel, Switzerland), according to the manufacturer's instructions. After extraction, DNA samples were kept at $4^{\circ} \mathrm{C}$ and RNA samples were stored at $-80^{\circ} \mathrm{C}$. DNA and RNA quantity was first measured with the Nanodrop 2000 spectrophotometer (Thermo Fisher Scientific, Waltham, MA, USA). When the total RNA yield was less than $0.1 \mu \mathrm{g}$, a second biopsy sample with a tumor percentage $\geq 50 \%$ - if available - was used for RNA isolation with the Qiagen RNeasy kit. Double-stranded DNA (dsDNA) quantity and RNA quality was analyzed on the Agilent 2100 Bioanalyzer (Agilent, Santa Clara, CA, USA). Currently, the most common method to assess RNA integrity is by calculating the RNA integrity number (RIN) from an electrophoretic trace. This number varies between 1 (totally degraded RNA) and 10 (intact RNA). DNA and RNA input recommendations for next-generation sequencing differ between protocols. dsDNA and RNA with little to no signs of degradation (RIN > 8) are preferably used. For the purpose of this study, the cut-off for a sufficient quantity of high-quality DNA was set at a minimum of $1.0 \mu \mathrm{g}$ of dsDNA. For RNA, this was at least $0.1 \mu \mathrm{g}$ of RNA with a RIN of at least 8 . These numbers are according to the requirements suggested in the Illumina Truseq (Illumina, San Diego, CA, USA) DNA and RNA sequencing library preparation protocols.

\section{Statistics}

Differences in the median RIN between samples with a different interval between sampling and freshfreezing were compared using the Kruskal-Wallis test. All tests were two sided and a $P$-value $<0.05$ was considered statistically significant. SPSS statistical software (version 20.0; SPSS, Chicago, IL, USA) was used for analysis.

\section{RESULTS}

\section{Included patients}

Between September 2008 and May 2013, endoscopic biopsy samples of 118 patients with esophageal cancer who were referred for potentially curative treatment were obtained according to the study protocol. Twenty-eight patients with squamous cell carcinoma, one patient with a neuroendocrine carcinoma and one patient with an undifferentiated carcinoma were excluded from the analysis. Of the remaining 88 patients diagnosed with adenocarcinoma, 10 patients appeared to have metastatic disease during further 


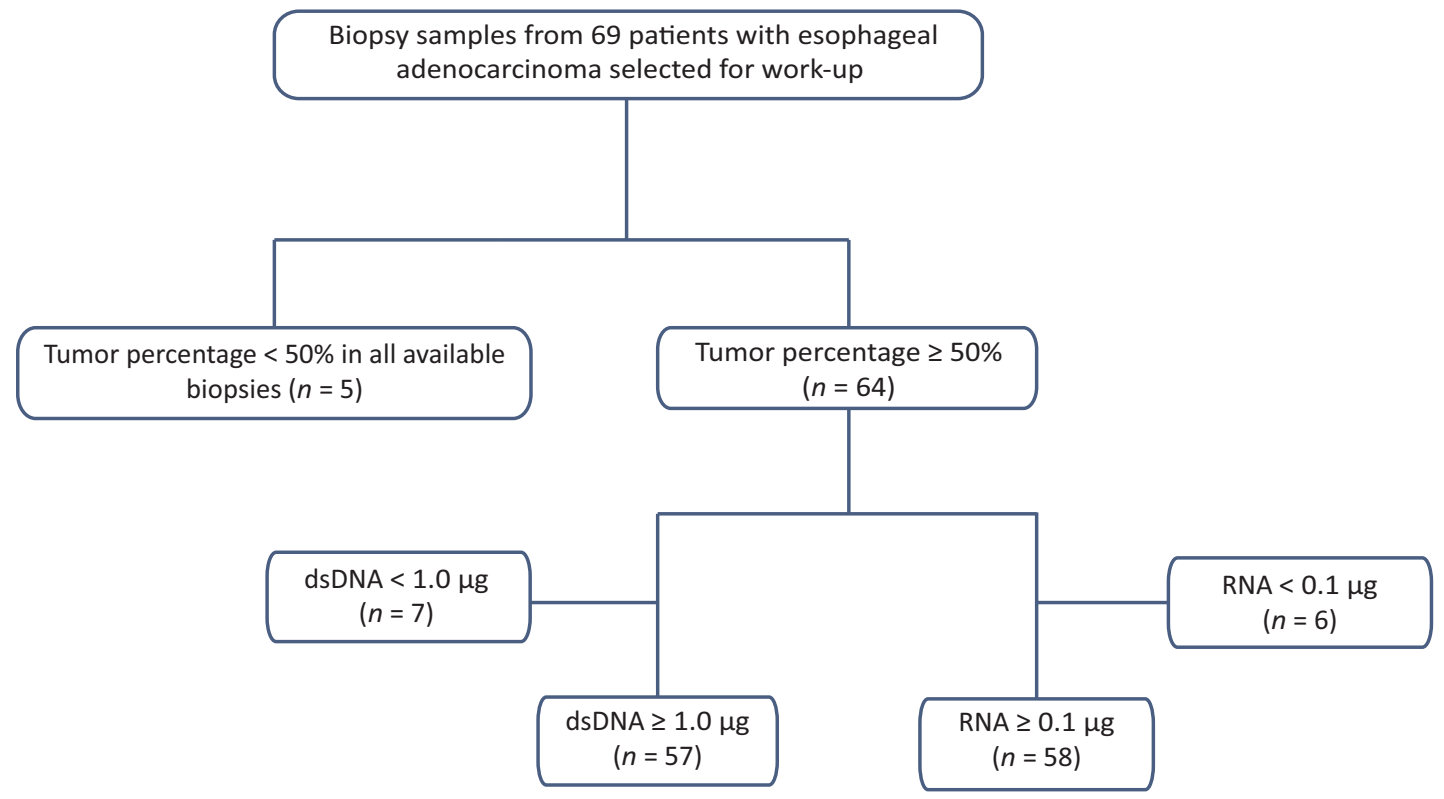

Fig. 1 Work-up of endoscopic biopsy samples from patients with esophageal adenocarcinoma who were treated with chemoradiotherapy followed by surgery. dsDNA, double-stranded DNA.

diagnostic work-up and were treated with palliative therapy. Seven patients were treated with definitive chemoradiotherapy. One patient did not complete chemoradiotherapy because of severe pulmonary complications. In another patient, fresh-frozen material had to be used for routine diagnostics. This left a homogeneous cohort of 69 patients with esophageal adenocarcinoma included in this study (Fig. 1). Of these, nine (13\%) were staged as cT2 and $60(87 \%)$ as cT3. Forty-nine $(71 \%)$ of 69 patients had clinically lymph node-positive disease. Twenty-one (30\%) of 69 tumors were moderately differentiated and $29(42 \%)$ had a poor differentiation grade. In $14(20 \%)$ of 69 tumors, the differentiation grade was unknown.

\section{Tumor percentage}

In 33 of 69 study patients (48\%), more than one tumor biopsy was required to obtain a sample with a tumor percentage of at least $50 \%$. In total, 129 biopsy samples were used in this study: one biopsy was sufficient for a tumor percentage $>50 \%$ in 36 patients $(52 \%)$, a second biopsy was necessary in 16 patients $(23 \%)$, a third biopsy in seven patients $(10 \%)$, and a fourth biopsy in five patients $(7 \%)$. In the endoscopic biopsies of another five patients (7\%), the tumor percentage was below $50 \%$ in all four samples. This left 64 samples that were suitable for subsequent DNA and RNA isolation. Low tumor percentages were mainly caused by normal epithelium, stromal tissue, and/or leukocyte infiltration (Fig. 2A-D).

\section{DNA/RNA quantity and quality}

DNA and RNA quantity and quality results are summarized in Table 1. First, a protocol was used that allows isolation of both DNA and RNA from the same sample (DNA/RNA AllPrep kit). In 57 of 64 samples $(89 \%)$, the dsDNA quantity was at least $1.0 \mu \mathrm{g}$. In 18 of 64 samples (28\%), RNA quantity was lower than $0.1 \mu \mathrm{g}$. In 12 of these patients, a second biopsy with a tumor percentage $\geq 50 \%$ was available. In all these samples, more than $0.1 \mu \mathrm{g}$ of RNA was isolated with the RNeasy kit. The median RIN of all 58 RNA samples with a yield higher than $0.1 \mu \mathrm{g}$ was 5.5 (range 1.8-8.9). Only 4 of 58 samples (7\%) had a RIN above 8 . Thirty samples $(52 \%)$ had a RIN between 5 and 8 , and in 24 samples (41\%), the RIN

Table 1 DNA and RNA quantity and quality using the Qiagen Allprep and RNeasy kits

\begin{tabular}{|c|c|c|}
\hline & $\begin{array}{l}\text { DNA/RNA } \\
\text { AllPrep kit } \\
(n=64)\end{array}$ & $\begin{array}{l}\text { RNEasy kit } \dagger \\
(n=12)\end{array}$ \\
\hline \multicolumn{3}{|l|}{ dsDNA } \\
\hline Concentration $(\mathrm{ng} / \mu \mathrm{L}) \S$ & $47(2-240) \ddagger$ & - \\
\hline Total yield $(\mu \mathrm{g}) \S$ & $2.4(0.1-12.0) \$$ & - \\
\hline $\begin{array}{c}\text { Number }(\%) \text { of samples } \\
\text { with yield }>1.0 \mu \mathrm{g} \S\end{array}$ & $57(89 \%)$ & - \\
\hline \multicolumn{3}{|l|}{ RNA } \\
\hline Concentration $(\mathrm{ng} / \mu \mathrm{L})$ & $33(1-808) *$ & $75(25-194)+$ \\
\hline Total yield $(\mu \mathrm{g}) \boldsymbol{T}$ & $0.5(0.01-11.0)+$ & $3.1(1.2-9.7) \ddagger$ \\
\hline $\begin{array}{c}\text { Number }(\%) \text { of samples } \\
\text { with yield }>0.1 \mu \mathrm{g}\end{array}$ & $46(72 \%)$ & $12(100 \%)$ \\
\hline RIN & $5.5(2.3-8.4) \ddagger$ & $5.7(1.8-8.9) \ddagger$ \\
\hline
\end{tabular}

$\dagger$ From 12 patients in whom the RNA yield was less than $0.1 \mu \mathrm{g}$ using the AllPrep kit, a second biopsy with sufficient tumor was available for RNA isolation using the RNEasy kit. \$Median (range). §Concentration and total yield according to the Agilent 2100 Bioanalyzer (Agilent, Santa Clara, CA, USA). $\uparrow$ Concentration and total yield according to the Nanodrop 2000 spectrophotometer (Thermo Fisher Scientific, Waltham, MA, USA). dsDNA, doublestranded DNA; RIN, RNA integrity number. 


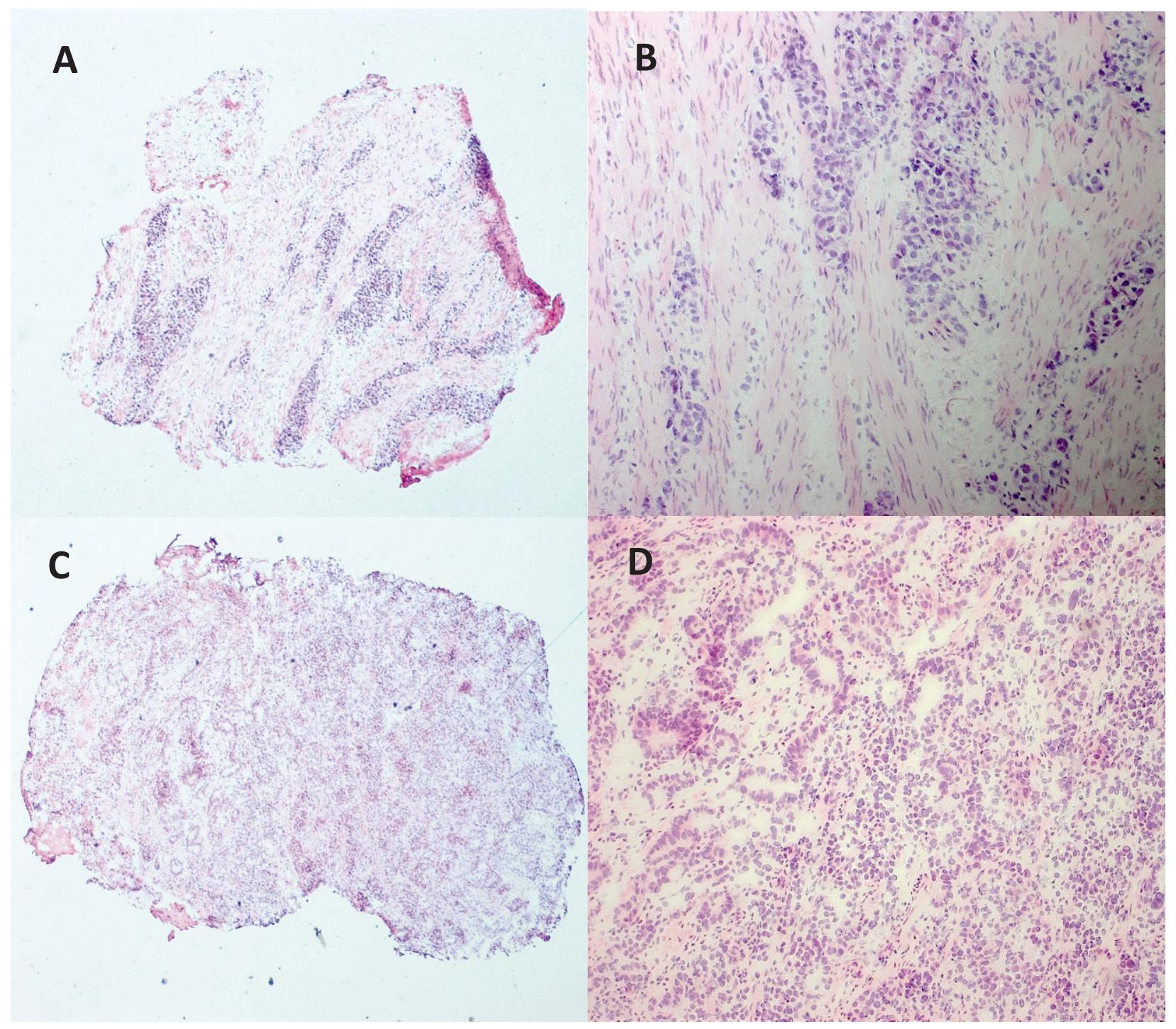

Fig. 2 Hematoxylin and eosin slides from study biopsies with normal stroma and leukocyte infiltration compromising the tumor percentage. (A, B; magnified): tumor biopsy with a considerable amount of normal stroma. (C, D; magnified): tumor biopsy with a considerable amount of leukocyte infiltration compromising the tumor percentage.

was lower than 5. From 52 samples, the time interval between biopsy sampling and fresh-freezing was available. RIN values decreased significantly with increased time periods before fresh-freezing of biopsy samples (Fig. 3). In 18 of 24 samples (75\%) frozen after 1 hour, the RIN was below 5 .

\section{DISCUSSION}

The current study results provide a detailed description of our prospective biobanking efforts with untreated esophageal cancer endoscopic biopsies. Several important issues were encountered that should be taken into account in future biobanking projects. Low tumor percentages in biopsy samples led to a considerable sample loss. In 5 of $69(7 \%)$ patients, the tumor percentage was below 50\% in all (four) endo(C) 2015 International Society for Diseases of the Esophagus scopic biopsy samples obtained for research purposes. In 57 of 64 samples $(89 \%)$ with a tumor percentage of at least $50 \%$, more than $1.0 \mu \mathrm{g}$ of dsDNA could be isolated. Sufficient RNA (at least $0.1 \mu \mathrm{g}$ ) was isolated from 58 of 64 samples $(91 \%)$. A time delay between sampling and fresh-freezing of biopsies had a negative effect on RNA quality: in 18 of 24 samples $(75 \%)$ frozen after 1 hour, RNA showed considerable degradation (RIN lower than 5).

\section{Tumor percentage}

When studying DNA from tumor tissue, these samples are preferably representative with an adequate number of tumor cells. Lower tumor percentages can significantly decrease the sensitivity of detecting genomic aberrations by diluting tumor DNA with DNA from normal tissue. This is exem- 


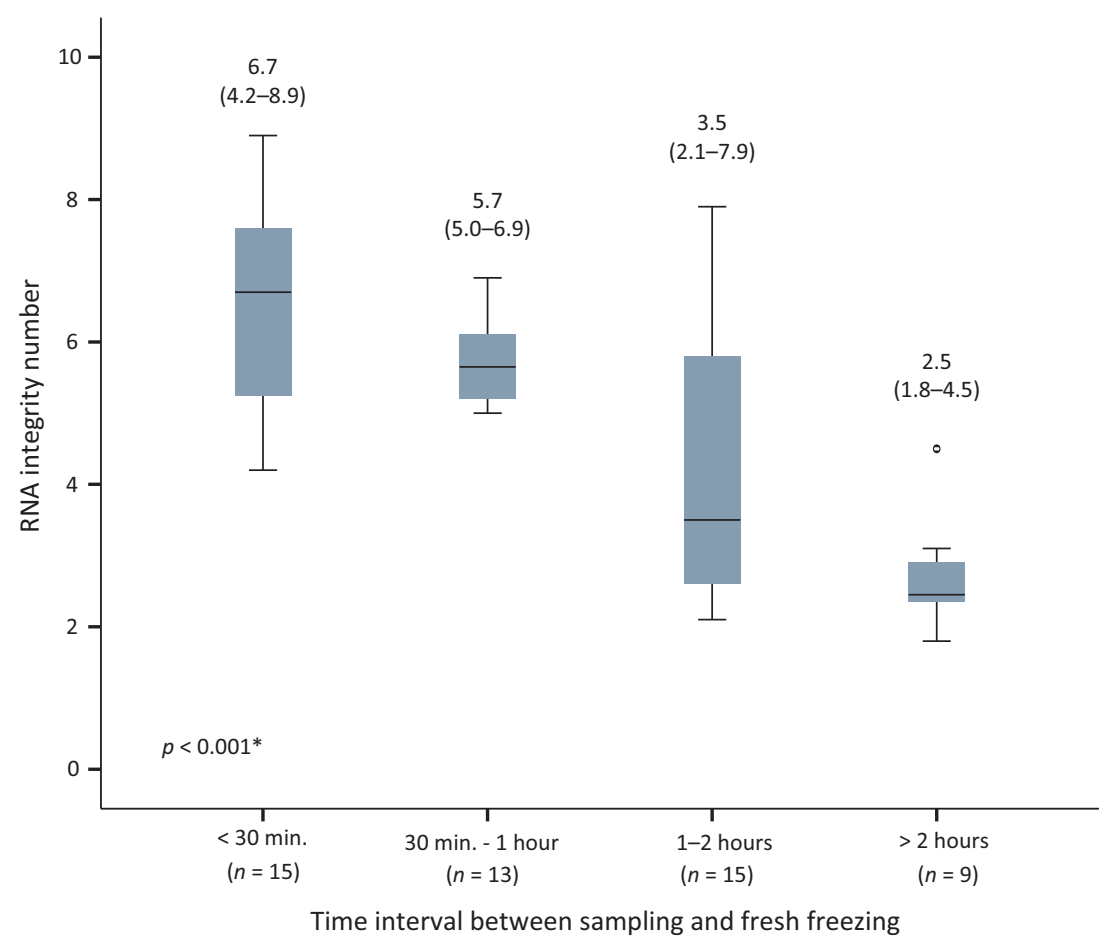

Fig. 3 Effect of a time interval between sampling and fresh-freezing on RNA quality of endoscopic biopsies of esophageal adenocarcinoma. Numbers above box plots display the median (range) RNA integrity number. RIN, RNA integrity number. *Kruskal-Wallis test.

plified in a study with colon cancer samples in which the ability to correctly identify KRAS mutations was diminished with a decreasing tumor percentage. ${ }^{13} \mathrm{In}$ contrast, the effect of normal tissue 'contamination' on RNA gene expression profiling has been questioned. In most studies aimed to develop prognostic gene expression signatures, only samples with a high $(>50 \%)$ percentage of tumor cells are allowed. ${ }^{14,15}$ However, in a study validating the 70 -gene breast cancer prognosis signature, the minimum tumor percentage was decreased to $30 \%$ as this figure also generated a reliable read-out. ${ }^{5}$ Consequently, no definite thresholds are defined but most in most studies samples with a minimum tumor percentage between 50 and $70 \%$ are used. Because of the introduction of neoadjuvant treatment strategies, untreated esophageal cancer tissue samples can only be obtained by endoscopic biopsy. The acquisition of representative tumor samples during endoscopy is more challenging than that of adequate material from surgical resection specimens, but methods to estimate the tumor percentage in esophageal biopsy samples vary widely in current literature. In some studies, the tumor percentage was assessed on each biopsy sample used for downstream applications, ${ }^{11,12}$ while in other studies an adjacent biopsy sample was used..$^{8-10}$ Our results underline the need to assess the tumor percentage in all biopsy samples used for downstream applications. Low tumor percentages in biopsy samples led to a considerable sample loss, emphasizing the need to obtain multiple biopsies. In the current study, we included patients with locally advanced esophageal adenocarcinoma. The majority had a clinical tumor stage of T3, with a moderate to poor tumor differentiation. Even in these advanced tumors, obtaining a representative biopsy sample was a challenge. In recent years, biomarker studies and the development of risk stratification models have increasingly focused on Barrett's associated pre-cancerous and early esophageal adenocarcinoma. ${ }^{16}$ It is likely that obtaining representative samples from these lesions is even more challenging. Moreover, given the inter- and intra-observer variability in correctly grading these lesions (e.g. low-grade vs. high-grade dysplasia), the role of an expert pathologist in biobanking project with these samples becomes even more important. ${ }^{17}$

\section{DNA/RNA quantity and quality}

Assessing the quantity and quality of DNA/RNA is pivotal in determining which protocol is used for preparing the samples for sequencing. Currently, intact dsDNA is needed for next-generation sequencing. For RNA sequencing, protocols allowing the input of minute quantities of degraded RNA have been developed, but sequencing results are still better with protocols requiring intact RNA. $^{18}$ Because archival formalin-fixed paraffin-embedded tissue usually yields degraded DNA and RNA, fresh-frozen specimens are preferred. In the current study, the majority 
of samples yielded a sufficient quantity $(>1.0 \mu \mathrm{g})$ intact dsDNA. In contrast, RNA was degraded in a large number of samples. Although it is generally believed that fresh tissue should be frozen as quickly as possible to prevent RNA degradation, there are some conflicting results in current literature. In two studies with resection specimens from colon and pancreatic cancer, a prolonged time period before freshfreezing did not adversely affect RNA quality.,4 These results are in contrast to a study by Hong et al., in which colorectal carcinoma biopsy samples obtained from resection specimens were left at $4^{\circ} \mathrm{C}$ for 10-90 minutes before fresh-freezing in liquid nitrogen. ${ }^{19}$ The mean RIN for samples frozen after 10 minutes was 7.5 compared with 4.2 for samples frozen after 90 minutes. Moreover, 18 of 20 samples $(90 \%)$ of samples frozen after 90 minutes had a RIN $<7$. In a study by Maher et al., 25 endoscopic biopsy samples from esophageal cancer patients were collected for gene expression analysis. ${ }^{10}$ They report that in 13 of 25 samples (52\%), RNA of sufficient quantity and quality was obtained for microarray analysis. In this study, biopsy samples were immediately placed in an RNA protective agent (RNAlater) before fresh-freezing. Taken together, a short time interval between tissue sampling and fresh-freezing seems especially important for small endoscopic biopsy samples.

The use of different protocols for DNA and RNA isolation adds complexity to the interpretation of the current literature on biobanking. Protocols allowing simultaneous isolation of DNA and RNA greatly increase the efficient use of valuable tissue, but the performance regarding DNA and RNA yield and quality in comparison with dedicated kits has been questioned. In a study by Mathieson et al., the AllPrep kit was compared with two dedicated kits for DNA (Puregene) and RNA (RNeasy) isolation..$^{20}$ The dedicated DNA isolation kit performed better than the AllPrep kit in yield and quality, but RNA yield and quality were comparable between the AllPrep and RNeasy kit. In the current study, the RNeasy kit was used when the total RNA yield with the AllPrep kit was $<0.1 \mu \mathrm{g}$ and another biopsy sample with an adequate tumor percentage was available. The RNA yield from these samples was higher, but RNA quality still differed considerably. It was not the goal of the current study to compare the efficiency of different kits and protocols and these results need to be interpreted with caution. Still, it is possible that the results of DNA and RNA isolation can be improved when dedicated kits are used, with the use of more tissue as a likely trade-off.

\section{Concluding remarks}

In conclusion, the results from this study show that obtaining high-quality DNA and RNA from endo(C) 2015 International Society for Diseases of the Esophagus scopic esophageal adenocarcinoma samples is feasible, but requires thorough logistical planning. Both the tumor percentage in biopsy samples and DNA/ RNA yield and quality emphasize the need for sampling multiple biopsies and minimizing the time delay before fresh-freezing in the biobanking process of esophageal adenocarcinoma.

\section{Acknowledgments}

The authors would like to thank Annegien Broeks and Ron Kerkhoven for critically reading and commenting on this manuscript.

\section{References}

1 Mardis E R. A decade's perspective on DNA sequencing technology. Nature 2011; 470: 198-203.

2 Barnes R O, Parisien M, Murphy L C, Watson P H. Influence of evolution in tumor biobanking on the interpretation of translational research. Cancer Epidemiol Biomarkers Prev 2008; 17: 3344-50.

3 Rudloff U, Bhanot U, Gerald W et al. Biobanking of human pancreas cancer tissue: impact of ex-vivo procurement times on RNA quality. Ann Surg Oncol 2010; 17: 2229-36.

4 Bao W G, Zhang X, Zhang J G et al. Biobanking of freshfrozen human colon tissues: impact of tissue ex-vivo ischemia times and storage periods on RNA Quality. Ann Surg Oncol 2012; 20: 1737-44.

5 Mook S, Bonnefoi H, Pruneri G et al. Daily clinical practice of fresh tumour tissue freezing and gene expression profiling; logistics pilot study preceding the MINDACT trial. Eur $\mathbf{J}$ Cancer 2009; 45: 1201-8.

6 Cunningham D, Allum W H, Stenning S P et al. Perioperative chemotherapy versus surgery alone for resectable gastroesophageal cancer. N Engl J Med 2006; 355: 11-20.

7 van Hagen P, Hulshof M C, van Lanschot J J et al. Preoperative chemoradiotherapy for esophageal or junctional cancer. N Engl J Med 2012; 366: 2074-84.

8 Luthra R, Wu T T, Luthra M G et al. Gene expression profiling of localized esophageal carcinomas: association with pathologic response to preoperative chemoradiation. J Clin Oncol 2006; 24: 259-67.

9 Motoori M, Takemasa I, Yamasaki M et al. Prediction of the response to chemotherapy in advanced esophageal cancer by gene expression profiling of biopsy samples. Int J Oncol 2010; 37: 1113-20.

10 Maher S G, Gillham C M, Duggan S P et al. Gene expression analysis of diagnostic biopsies predicts pathological response to neoadjuvant chemoradiotherapy of esophageal cancer. Ann Surg 2009; 250: 729-37.

11 Duong C, Greenawalt D M, Kowalczyk A et al. Pretreatment gene expression profiles can be used to predict response to neoadjuvant chemoradiotherapy in esophageal cancer. Ann Surg Oncol 2007; 14: 3602-9.

12 Schauer M, Janssen K P, Rimkus C et al. Microarray-based response prediction in esophageal adenocarcinoma. Clin Cancer Res 2010; 16: 330-7.

13 Dijkstra J R, Heideman D A, Meijer G A et al. KRAS mutation analysis on low percentage of colon cancer cells: the importance of quality assurance. Virchows Arch 2013; 462: 39-46.

$14 \mathrm{t}$ Veer L J, Dai H, van de Vijver M J et al. Gene expression profiling predicts clinical outcome of breast cancer. Nature 2002; 415: 530-6.

15 Peters C J, Rees J R, Hardwick R H et al. A 4-gene signature predicts survival of patients with resected adenocarcinoma of the esophagus, junction, and gastric cardia. Gastroenterology 2010; 139: 1995-2004.

16 Zeki S, Fitzgerald R C. The use of molecular markers in predicting dysplasia and guiding treatment. Best Pract Res Clin Gastroenterol 2015; 29: 113-24. 
17 Goldblum J R. Current issues in Barrett's esophagus and Barrett's-related dysplasia. Mod Pathol 2015; 28 (Suppl. 1): S1-6.

18 Sun Z, Asmann Y W, Nair A et al. Impact of library preparation on downstream analysis and interpretation of RNA-Seq data: comparison between Illumina PolyA and NuGEN Ovation protocol. PLoS ONE 2013; 8: e71745.
19 Hong S H, Baek H A, Jang K Y et al. Effects of delay in the snap freezing of colorectal cancer tissues on the quality of DNA and RNA. J Korean Soc Coloproctol 2010; 26: 31623.

20 Mathieson W, Thomas G A. Simultaneously extracting DNA, RNA, and protein using kits: is sample quantity or quality prejudiced? Anal Biochem 2013; 433: 10-8. 